\title{
A mobile biosensor to detect cardiorespiratory activity for stress tracking
}

\author{
Nicola Carbonaro, Alessandro Tognetti, Gaetano \\ Anania, Danilo De Rossi \\ Research Center "E.Piaggio", Faculty of Engineering, \\ University of Pisa \\ nicola.carbonaro@centropiaggio.unipi.it
}

\author{
Pietro Cipresso, Andrea Gaggioli, Giuseppe Riva \\ Applied Technology for Neuro-Psychology Lab \\ IRCCS Istituto Auxologico Italiano \\ Milan, Italy
}

Stress is an increasingly recognized phenomenon that has negative effects on growing numbers of people. Stress assessment is a complex issue, but different studies have shown that monitoring user psychophysiological parameter during daily life can be greatly helpful in stress evaluation. In this study a wearable biosensor platform able to collect physiological and behavioral parameters is reported. The developed wearable platform, in terms of hardware and processing algorithms, is described. Moreover the use of this wearable biosensor platform in combination with advanced simulation technologies, such as virtual reality offer interesting opportunities for innovative personal health-care solutions to stress. A recently founded European project, "INTERSTRESS - Interreality in the management and treatment of stress-related disorders," will take into account these relevant aspects.

Keywords: Stress, Psychophysiology, Mobile biosensor, Electrocardiogram, Respiration signal, Bio-behavioral Methods.

\section{INTRODUCTION}

Research interest in psychological stress and its cognitive and bodily responses has been growing over the last decades.

Stress is an increasingly recognized phenomenon that has negative effects on growing numbers of people [1,2]. Chronic stress is responsible for premature mortality in Western countries, and work-related stress accounts for premature cardiovascular mortality rates.

Within this panorama, it is easily understandable that stress assessment is a complex issue independently to instruments, biosensors or psychological method used for the assessment [3].

Several studies showed interesting results that support the feasibility of detecting affective states through psychophysiological data acquisition and analysis [4-5]. The affective computing group at MIT, led by Rosalind Picard, published several research studies that highlighted the use of psychophysiological measures to deduce and classify emotional states while study participants were performing different kinds of PC activities [6-7].

On the other hand, there are few studies that has tested the feasibility of such platform to actually elicit affective states. This consideration needs to be reviewed further to understand its implications fully for behavioral health care. For example, in a therapy for a stress-related disorder in a clinical setting is essential to elicit an affective state. In fact, standard cognitive behavioral therapies, such as biofeedback and relaxation, work to modify affective states through direct elicitation of positive emotions or a stressful situation that the subject progressively then learns (or has already learned) to manage.

Researchers highlighted in particular the usefulness of wearable biosensors used in mobile platforms [8,9] and Virtual Reality environments [10-13] or both [14] to detect changes in the physiological and affective states. This can be essential, for example in mobile clinical setting, to share that information with the subject and eventually also interested caregivers, such as professional medical staff, relatives, or even friends.

This study reports the development of a novel wireless biomonitoring system for the continuous tracking of physiological and behavioural user parameters that used in combination with technological-based solutions, such as virtual reality and mobile phone, could offer interesting opportunities for innovative personal health-care solutions to stress .

\section{METHODS}

The Personal Biomonitoring System (PBS) is a platform that is conceived to be available to the patient during daily activities to collect, fuse and analyze patient behavior and his general and physiological status. This wireless biomonitoring system unobtrusively performs a real-time monitoring of heart rate (HR), heart rate variability (HRV) and breathing rate (BR), as meaningful physiological parameters to study the stress correlation. Moreover the PBS carries out a continuous tracking of activity level and posture of the user, as behavioral parameter for patient contextual identification.

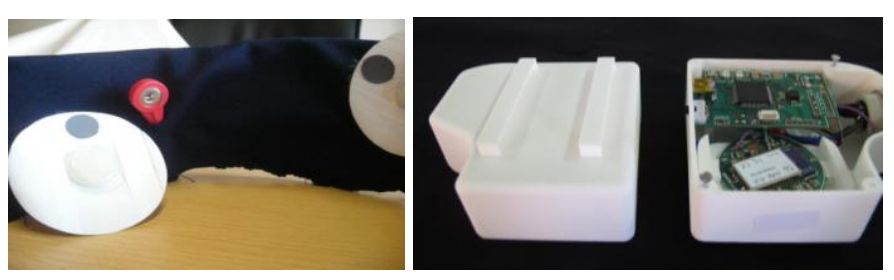

Fig. 1 The chest band realized and its electronic device

Data elaborated are sent to a personal data assistant (PDA) which will perform a provisional stress analysis useful to 
trigger a more accurate analysis connecting to a central database or to perform a local biofeedback strategy.

\section{A. Hardware description}

Different modules are embedded in the PBS. Each component design was oriented to usability and user comfort, without leaving out the importance to achieve reliable stress-related parameters. The electrodes, positioned in the chest, are embedded in the elastic band of Fig. 1a, providing a portable device in a all-in-one solution for physiological (cardiac and breathing monitoring) and behavioral data acquisition.

Fig. 2 shows the three different subsystems embedded in the PBS platform: i) the HUB collects all extracted parameters and send them to the PDA via Bluetooth. The HUB is the master node of the internal network, coordinating the exchange of data with the ECG module and BR module, respectively. Moreover, the HUB provides the power supply of the whole system and includes also the circuitry for battery charge management.

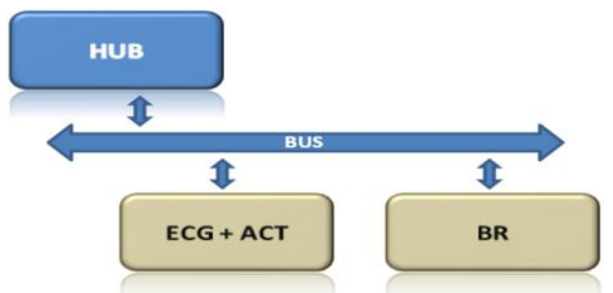

Fig. 2 Block diagram of PBS system

ii) $\mathrm{ECG}+\mathrm{ACT}$ : this is the subsystem devoted to the extraction of the cardiac parameter, the heart rate (HR) and heart rate variability (HRV), and relevant information of user activity (ACT). iii) The last subsystem is the one for the extraction of the breathing rate parameter (BR).

All these subsystems will be analyzed in detail in the next sections.

\section{ECG system}

The PBS ECG block is a 3 leads ECG sensor that samples signals at the frequency of $256 \mathrm{~Hz}$. The front-end is based on the INA321 instrumentation amplifier that simply cancels out the common-mode and amplifies the input differential ECG signal to about $5 x$ (no external $R G$ is added).

The signal at the output of INA321 is further amplified by one of the three integrated operational amplifiers available in the microcontroller (MSP family made by Texas Instruments, MSP430FG439), to reach the total 500x amplification.

The second integrated operational amplifier is used to manage the variation of the $\mathrm{dc}$ content of the differential output signal, popularly called baseline wandering. This amplifier is designed as an analog integrator scheme that feeds back the integration of the dc content of the 5x amplified ECG to the INA321.

The third integrated operational amplifier is used for the active drive of the reference electrode.

The amplified ECG signal is internally digitized using the on-chip analog-to-digital converter available in the microcontroller. The core of the system, is the low power microcontroller that elaborates and analyzes raw sensor data and extracts HR and HRV parameters directly on board.

\section{BR system}

There is substantial evidence that alterations in respiratory rate can be used to predict potentially serious clinical events such as cardiac arrest or admission to the intensive care unit $[15,16]$.

Indirect techniques that can be implemented in wearable systems are respiratory inductive plethysmography [17], impedance plethysmography [18], piezoresistive pneumography [19], and/or piezoelectric pneumography. These systems are minimally invasive and do not interfere with physical activity, but most of them suffer from motion artifact especially if movements are at the thorax level. Considering this, a new sensor configuration was adopted. This new configuration consists of using a piezoelectric cable, made of Polyvinylidene (PVDF), integrated in the chest band in order to increase the Signal to Noise Ratio (SNR) making the breathing signal detector reliable and robust. The working principle is to measure mechanical forces due to the chest movements and correlate them to the fundamental frequency of respiration activity.
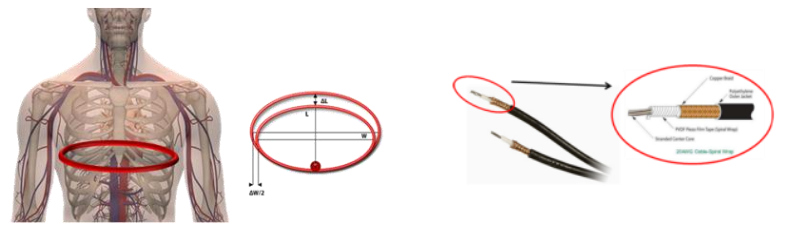

Fig. 3 Representation of piezo cable application. The cable size is 20 AWG, and the typical piezoelectric capacitance and sensitivity of the cable are $650 \mathrm{pFm}-1$ and $20 \mathrm{pCN}-1$, respectively.

When the cable is compressed or stretched, a charge is generated between the centre core and the outer braid shield.

The charge of the sensor is converted into a proportional voltage by a current-voltage converter, and then the output signal is fed to an A/D converter unit, embedded internally in the microcontroller. The charge-voltage converter is constructed using an OPA124 by Texas Instrument.

\section{Activity system}

In this study, behavioral information are not considered as stress indicators but they will be used as context for physiological measures.

According to this point the well known signal magnitude area (SMA) index is extracted from a tri-axial accelerometer integrated in the cardiac module, in order to discriminate the level of activity the user performed [20][21].

The ADXL330 produced by Analog Device was selected for its main characteristic such as small size, thin, low power, complete 3 -axis accelerometer all on a single monolithic IC. 


\section{B. Algorithm and features extraction}

\section{QRS detection, HR and HRV extraction}

An ECG analysis algorithm was developed to elaborate and process signals generated from the wearable PBS. QRS complex needs to be detected to generate heart rate and RR intervals. Thus, an accurate QRS detector is important in order to extract reliable $\mathrm{HR}$ or RR intervals to make meaningful HRV analysis and mental stress correlation. However, an accurate QRS complex detection may be difficult due to the physiological variability of the QRS complex and various types of noise that can be present in the ECG signal.

Typical noise artifacts in ECG signals are power line interference, electrode contact noise, motion artifacts, and baseline drift [22]. Motion artifact is the most relevant in case of wearable monitoring systems used out of clinics during activity of daily living, where it is important to measure physiological signals accurately anytime and anywhere. Thus, a novel algorithm for robust and real-time HR and HRV extraction from ECG signal was developed in order to extract further correlates to mental stress [23] .

There are numerous detection methods for QRS complexes including Pan-Tompkins method [24], wavelet [25] and Hilbert [26] transforms. Moreover, morphological approaches were developed for baseline correction and noise suppression in clinical ECG signals [27]. The algorithm developed, starting from the original Pan-Tompkins idea, uses a Kalman filter to extract reliable QRS complex, reducing the noise in the ECG signal, without applying any threshold and with very low computational cost.

The novelty of the algorithm is the combination of the Kalman filter with the predictor stage. With respect to the different algorithms present in literature that use high order low pass filter on the raw ECG signal, low time delay or amplitude attenuation are added applying the Kalman filter. Moreover, the design of the predictor stage based on assessment of the future QRS parameters allows to have $\mathrm{R}$ peaks and RR time distance evaluations independent from the inter-subject variability and from the measurements system used [23].

\section{BR monitoring}

The breathing rate detection algorithm is executed in real time on the module low power micro-controller (MSP430F149 by Texas Instruments). The routine computes a real time peak recognition function of the breathing signal, not using any threshold value or empiric rules.

A previous study has demonstrated how this fundamental frequency is a good approximation of the subject BR [28]. The choice of the PVDF satisfies the requirements of reliability, reproducibility, and high sensitivity in the human temperature range. The sensor configuration (see Fig. 3Fig. 3) permitted to obtain low contribution of the movement artifact and a maximum contribution of the signal, which is generated by the chest pressure applied to the sensor [29]. This configuration makes the breathing signal detector reliable and robust also during user movements [28] .

\section{Activity elaboration}

The accelerometer measures the acceleration and local gravity that it experiences. Considering a calibrated tri-axial accelerometer (i.e. offset and sensitivity are compensated and the output is expressed in unit of g), the accelerometer signal (y) contains two factors: one is due to the gravity vector $(\mathrm{g})$ and the other is due to the system inertial acceleration (a), both of them expressed in the accelerometer reference frame [30]:

$$
\mathbf{y}=\mathbf{a}-\mathbf{g} \quad\left(\begin{array}{l}
y_{1} \\
y_{2} \\
y_{3}
\end{array}\right)=\left(\begin{array}{l}
a_{1} \\
a_{2} \\
a_{3}
\end{array}\right)-\left(\begin{array}{l}
g_{1} \\
g_{2} \\
g_{3}
\end{array}\right)
$$

In static conditions only the factor due to gravity is present and the inclination of the accelerometer with respect to the vertical is known. In dynamic conditions, an estimation of the inclination is unreliable simply by using the raw accelerometer signal since the inertial acceleration is added to the gravity factor. This estimation error gets more important as the subject performs faster movements (e.g, running, jumping).

In order to estimate the activity intensity, the Signal Magnitude Area (SMA) is extracted from the inertial acceleration components detected by the worn sensor. The SMA is equal to the sum of the axis acceleration magnitude summations over a time window and normalized by the window length [31]. The SMA discrete form is given by:

$$
S M A(k)=\frac{1}{N}\left(\sum_{N k}^{N(k+1)-1}\left|a_{1}\right|+\sum_{N k}^{N(k+1)-1}\left|a_{2}\right|+\sum_{N k}^{N(k+1)-1}\left|a_{3}\right|\right)
$$

where $\mathrm{N}$ is the window length and (a1, a2, a3) are the three components of the inertial acceleration estimated by the accelerometer signal. At each time, the last available SMA value can be used to understand if the subject is resting, performing mild activities (among which walking) or intense activities (among which running). Considering the gravity component $g$ as a slowly varying one, the inertial component a can be approximated by means of a third order IIR high-pass digital filter with a cut-off frequency of $0.3 \mathrm{~Hz}$ and applied to the y components. This technique has been proved to be effective in order to classify the activity level [32].

Moreover the algorithm showed very good capabilities in distinguishing activity level performed by 9 subject during more than 5 hours of acquisition.

\section{TEST AND RESULTS}

Different experiments were performed in laboratory setting in order to test and validate the capability of each subsystem to perform robust and reliable extraction of the selected parameters.

Considering the behavioral user information, the algorithm showed very good capabilities in distinguishing activity level performed by 9 subject during more than 5 hours of 
acquisition. The good performance of the algorithm is reported in the Fig. 4, where the algorithm accurately classifies user activity between three different class of activity level ("none", "mild", "intense").

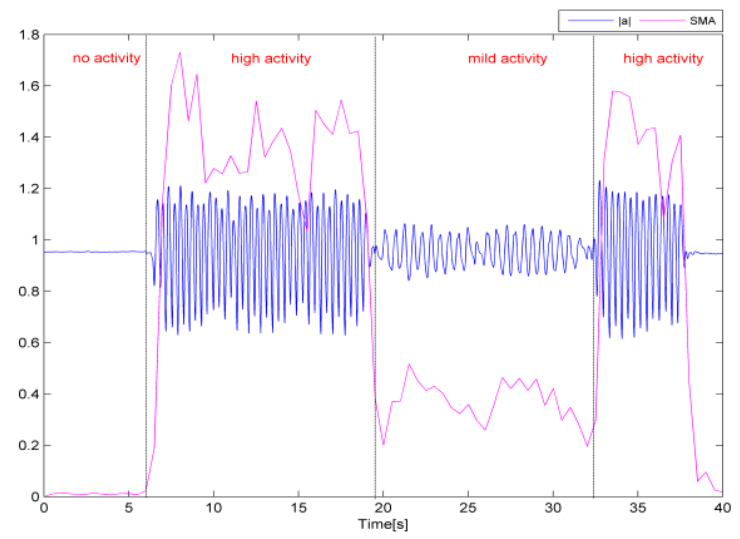

Fig. 4 Behavioral user classification. The blu signal represents the module of the acceleration signal, while the pink one is the SMA value that allows user activity discrimination.

Regarding ECG modules, the algorithm developed allows to have a $R$ peaks and RR time distance evaluations independent from the inter-subject variability and baseline wandering. In fact, in respect to the different algorithms present in literature that use high order low pass filter on the raw ECG signal, no time delay or amplitude attenuation are added applying the Kalman filter. Moreover, the filtering technique and the QRS complex detection are adaptive both to ECG signal, related to inter-subject variability, and to the measurement system, related to artifact noise. The method has been tested on all the MIT-BIH available records and showed very good results in terms of Sensitivity ( $\mathrm{Se}=99.95)$. Furthermore, several tests were carried out in order to analyse the capability of the realized hardware to acquire and elaborate real ECG signal.
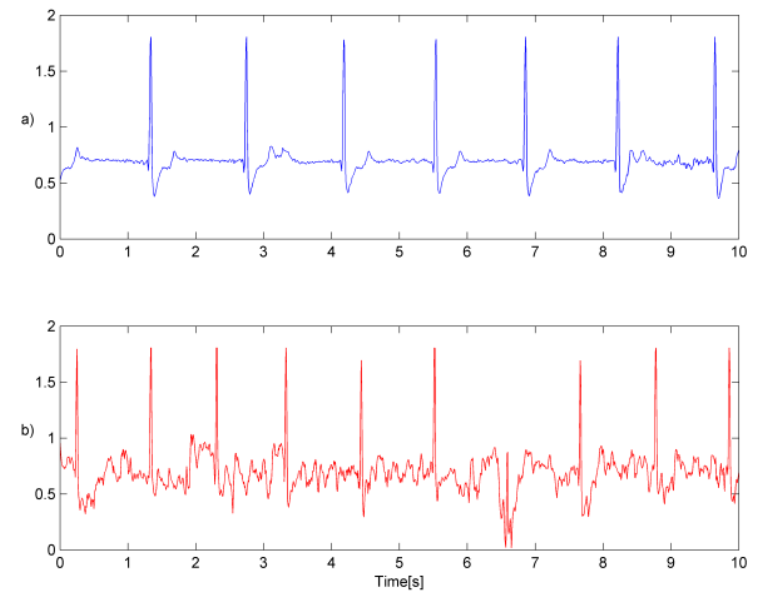

Fig. 5 ECG acquired using the PBS ECG block. In a) the user was asked to wear the system in a resting condition, while in b) the user was performing mild activities, such as walking or doing office activities.
It is possible to underline that Fig. 5 shows the PBS good capabilities to carefully reconstruct the typical ECG waveforms. Moreover, Fig. 5 b) points out the ability of the system to follow baseline variation due to motion artifact and to accurately reproduce cardiac peculiarity waves (QRS complex). The proved signal quality, reached also during motion activity, allows to extract reliable and robust meaningful cardiac parameters.

Regarding BR analysis, the choice of the PVDF satisfies the requirements of reliability, reproducibility, and high sensitivity in human temperature range. The sensor configuration permitted to obtain low contribution of movement artifact and a maximum contribution of the signal, which is generated by the chest pressure applied to the sensor . In Fig. 6 the signal acquired by the PBS BR block is reported. In particular it is represented the tracking of the user breathing signal and the real-time evaluation of its respiratory rate in resting condition, Fig. 6 a), and during fast respiration activity, Fig. 6 b).
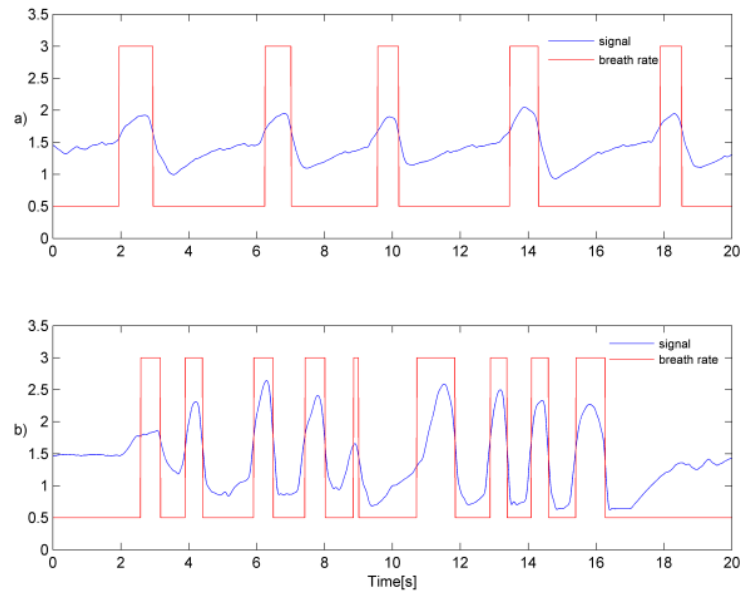

Fig. 6 Breathing Rate evaluation. The blu line represents the acquired breathing signal and the red one the recognition of the peak level useful for the evaluation of the breathing rate.

\section{DISCUSSION AND CONCLUSIONS}

The implementation of INTERSTRESS will be based on a database to collect physiological signals (in particular HRV measures) and psychological questionnaire during several participants' sessions. These sessions will also provide biofeedback sections with an advanced integration between the biosensors here presented and the Virtual Reality environments used during the participants' training.

Cognitive Behavioral (CBT) approach can be considered one of the best validated approach for stress management and stress treatment. Even if CBT is the treatment of choice for psychological stress, there is still room for improvement.

In particular new paradigm based on cutting-edge e-health can better fit to the needs of specific situations that stressed people need to consider.

Riva and coll. [33-34] recently introduced a new paradigm for e-health - "Interreality" - that integrates assessment and treatment within a hybrid environment, bridging physical and 
virtual world. By creating a bridge between virtual and real worlds, Interreality allows a full-time closed-loop approach actually missing in current approaches to the assessment and treatment of psychological stress: first, the assessment is conducted continuously throughout the virtual and real experiences: it enables tracking of the individual's psychophysiological status over time in the context of a realistic task challenge; second, the information is constantly used to improve both the appraisal and the coping skills of the patient: it creates a conditioned association between effective performance state and task execution behaviors. The potential advantages offered to stress treatments by this approach are: (a) an extended sense of presence: Interreality uses advanced simulations (virtual experiences) to transform health guidelines and provisions in experience; (b) an extended sense of community: Interreality provides social support in both real and virtual worlds; (c) a real-time feedback between physical and virtual worlds: Interreality uses bio and activity sensors and devices (PDAs, smartphones) both to track in real time the behavior and the health status of the user and to provide suggestions and guidelines.

Within this framework the biosensors here presented can meaningfully be considered as one of the few that totally integrates with all the virtual environment (VE) developed with NeuroVR (www.neurovr.org), a free software that allows everyone to create and to use VE for clinical application, including stress protocol [35].

These virtual environments are used in clinical setting and are fully controlled by the clinicians. These virtual worlds uses the sense of presence provided by the engaging virtual experience to practice several stress management exercises: relaxation techniques; VR biofeedback, assertiveness training, time management training, and problem-solving training.

The devices are integrated around two subsystems: the Clinical Platform (in patient treatment, fully controlled by the therapist) and the Personal Mobile Platform (real world support, available to the patient and connected to the therapist) that allow a) monitoring of the patient behavior and of his general and psychological status, early detection of symptoms of critical evolutions and timely activation of feedbacks in a closed loop approach; b) Monitoring of the response of the patient to the treatment, management of the treatment and support to the therapists in their therapeutic decisions.

The clinical use of these technologies in the Interreality paradigm is based on a closed-loop concept that involves the use of technology for assessing, adjusting and/or modulating the emotional regulation of the patient, his/her coping skills and appraisal of the environment (both virtual, under the control of a clinician, and real, facing actual stimuli) based upon a comparison of that patient's behavioural and physiological responses with a baseline or performance criterion.

In this study we considered Psychophysiological aspects for the management and treatment of stress-related disorders. One of the widely used instrument to analyze these aspects is the electrocardiogram (ECG) and, widely, the cardiorespiratory activity. In particular the Heart Rate Variability (HRV) measures have been generally used, giving evidences that lower variability seems to be higher related to negative moods, such us stress. A recently founded European project, "INTERSTRESS - Interreality in the management and treatment of stress-related disorders", will take in account these aspects, verifying ECG and HRV, in the interreality paradigm, i.e. creating a bridge between the physical and virtual worlds. In particular "stress detection" through ECG deserve an important consideration: there aren't empirical evidences to exactly discern strass-state components; in these aspects INTERSTRESS project will play an interesting role: the elicitations through hybrid environment could be a good chance to better discriminate a part of these components.

\section{ACKNOWLEDGMENTS}

The present work was supported by the European funded project "Interstress" - Interreality in the management and treatment of stress-related disorders (FP7- 247685).

\section{REFERENCES}

[1] Cipresso P., Gaggioli A, Serino S, Riva G (2012). Stress diffusion through complex networks. International Journal of Adaptive, Resilient and Autonomic Systems, vol. 3; p. 46-64, ISSN: 1947-9220, doi: 10.4018/jaras.2012010103

[2] Villani D, Grassi A, Cognetta C, Toniolo D, Cipresso P., Riva G (in press). Self-Help Stress Management Training Through Mobile Phones: An experience with oncology nurses. Psychological Services, ISSN: 1541-1559, doi: 10.1037/a0026459

[3] Cipresso P., Gaggioli A, Serino S, Pallavicini F, Raspelli S, Grassi A, Riva G (2012). EEG alpha asymmetry in virtual environments for the assessment of stress-related disorders. Studies In Health Technology And Informatics, vol. 173; p. 102-104, doi: 10.3233/978-1-61499-022-2102

[4] Magagnin V, Mauri M, CIPRESSO P., Mainardi L, Brown En, Cerutti S, Villamira M, Barbieri R (2010). Heart Rate Variability and Respiratory Sinus Arrhythmia Assessment of Affective States by Bivariate Autoregressive Spectral Analysis. Computers In Cardiology, vol. 37; p. 145-148.

[5] Giakoumis D, Drosou A, Cipresso P, Tzovaras D, Hassapis G, Gaggioli A, Riva G (2012), Using Activity-Related Behavioural Features towards more Effective Automatic Stress Detection, PLoS ONE 7(9): e43571; doi:10.1371/journal.pone.0043571

[6] Picard, Rosalind W. Affective computing. MIT press, 2000.

[7] Mauri M, Magagnin V, CIPRESSO P., Mainardi L, Brown En, Cerutti S, Villamira M, Barbieri R (2010). Psychophysiological signals associated with affective states. In: Engineering in Medicine and Biology Society (EMBC), 2010 Annual International Conference of the IEEE. Buenos Aires, Aug. 31 - Sept. 4 2010, p. 3563-3566. Doi: 10.1109/IEMBS.2010.5627465

[8] Cipresso P., Serino S, Villani D, Repetto C, Sellitti L, Albani G, Mauro A, Gaggioli A, Riva G (2012). Is your phone so smart to affect your state? An exploratory study based on psychophysiological measures. Neurocomputing, vol. 84; p. 23-30, doi: 10.1016/j.neucom.2011.12.027

[9] Villani D, Grassi A, Cognetta C, Cipresso P., Toniolo D, Riva G (2012). The Effects of a Mobile Stress Management Protocol on Nurses Working with Cancer Patients: a Preliminary Controlled Study. Studies In Health Technology And Informatics, vol. 173; p. 524-528, doi: 10.3233/978-1-61499-022-2-524

[10] Villani D, Repetto C, Cipresso P., Riva G (in press). May I experience more presence in doing the same thing in virtual reality than in reality? An answer from a simulated job interview. Interacting With Computers, 24(4): 265-272; doi: 10.1016/j.intcom.2012.04.008

[11] Raspelli S, Pallavicini F, Carelli L, Morganti F, Pedroli E, Cipresso P., Poletti B, Corra B, Sangalli D, Silani V, Riva G (2012). Validating the Neuro VR-based virtual version of the Multiple Errands Test: 
preliminary results. Presence-Teleoperators And Virtual Environments, vol. 21; p. 31-42, doi: 10.1162/PRES_a_00077

[12] Pallavicini F, Cipresso P., Raspelli S, Grassi A, Serino S, Vigna C, Triberti S, Villamira M, Gaggioli A, Riva G (2013). Is Virtual Reality Always an Effective Stressors for Exposure Treatments? Some Insights from a Controlled Trial. BMC Psychiatry, vol. 13:52, doi: 10.1186/1471-244X-13-52

[13] Cipresso P., La Paglia F., La Cascia C., Riva G., Albani G., La Barbera D. (in stampa). Break in volition: A virtual reality study in patients with obsessive-compulsive disorder. Experimental Brain Research. Doi: 10.1007/s00221-013-3471-y

[14] Repetto C, Gaggioli A, Pallavicini F, Cipresso P., Raspelli S, Riva G (2013). Virtual reality and mobile phones in the treatment of generalized anxiety disorders: a phase-2 clinical trial. Personal And Ubiquitous Computing, 17: 2, pp. 253-260 February, doi: 10.1007/s00779-0110467-0

[15] Fieselmann JF, Hendryx MS, Helms CM, et al. "Respiratory rate predicts cardiopulmonary arrest for internal medicine patients". J Gen Intern Med 1993;8:354-360.

[16] F.Q. AL-Khalidi, R. Saatchi,D. Burke,H. Elphick, and S. Tan, "Respiration Rate Monitoring Methods: A Review", Pediatric Pulmonology 46:523-529 (2011).

[17] J.S. Milledge, F.D. Stott, "Inductive plethysmography - a new respiratory transducer", J Physiol (Lond), 267:4, 1977.

[18] E.J.C. de Geus, G.H.M. Willemsen, C.H.A.M. Klaver, L. J.P. van Doornen, "Ambulatory measurement of respiratory sinus arrhythmia and respiration rate", Biological Psychology, 41, 205-227, 1995.

[19] D. De Rossi, F. Carpi, F.Lorussi, A.Mazzoldi, R.Paradiso, E.P. Scilingo, A. Tognetti, "Electroactive Fabrics and Wearable Biomonitoring Devices", AUTEX Research Journal, Vol. 3, No4, December, 2003.

[20] H.J. Luinge, P.H. Veltink, "Inclination Measurement of Human Movement Using a 3-D Accelerometer With Autocalibration", IEEE Trans. on Neural System and Rehabilitation Engineering, Vol.12, No.1, pp.112-121. March 2004.

[21] C. V. C. Bouten, K. T. M. Koekkoek, M. Verduin, R. Kodde, J. D. Janssen, "A Triaxial Accelerometer and Portable Data Processing Unit for the Assessment of Daily Physical Ac-tivity", IEEE Trans. on Biomedical Engineering, Vol.44, No. 3, March, 1997.

[22] G.M. Friesen, T.C. Jannett, M.A. Jadallah, S.L. Yates, S.R. Quint, and H.T. Nagle, "A Comparison of the Noise Sensitivity of Nine QRS Detection Algorithms", IEEE Trans. Biomed. Eng., January 1990, Vol. 37, No. 1, pp. 85-98.

[23] Carbonaro N. ,Anania G.,Dalle Mura G., Tesconi M., Tognetti, A., Zupone, G., De Rossi, D. , "Wearable biomonitoring system for stress management: A preliminary study on robust ECG signal processing,"
2011 IEEE International Symposium on a World of Wireless, Mobile and Multimedia Networks (WoWMoM), pp.1-6, 20-24 June 2011.

[24] J. Pan, W. Tompkin, "A real-time QRS detection algorithm". IEEE Trans Eng Biomed Eng 1985;32(3): 230-236.

[25] S. W. Chen, H. C. Chen and H. L. Chan, "A real time QRS detection method based on moving-averaging incorporating with wavelet denoising", Comp. Methods and Progs. In Biomed., vol. 82, 2006, pp. $187-195$

[26] D.S. Benitez, P.A. Gaydecki, A. Zaidi and A. P. Fitzpatrick, "A new QRS detection algorithm based on the hilbert transform", Computer in Cardiology, 2000 IEEE 27:379-382.

[27] Y.Sun, K.L.Chan, and S.M. Krishnan, "ECG Signal Conditioning by Morphological Filtering", Computers in Biology and Medicine, November 2002, Vol. 32, No. 6, pp. 465-479.

[28] A. Lanatà, E. P. Scilingo, E. Nardini, G. Loriga, R. Paradiso, and D. De Rossi, "Comparative evaluation of susceptibility to motion artifact in different wearable systems for monitoring respiratory rate," IEEE Trans. Inf. Technol. Biomed., vol. 14, no. 2, pp. 378-386, Mar. 2010.

[29] http://www.meas-spec.com/product/t product.aspx?id=2476\#.

[30] D. M. Karantonis, M. R. Narayanan, M. Mathie, N. H. Lovell, B. G. Celler, "Implementation of a Real-Time Human Activity Classifier Using a Triaxial Accelerometer for Ambulatory Monitoring", IEEE Trans. On Information Technology In Biomedicine, Vol.10, No.1, 2006.

[31] D. Curone, A. Tognetti, E. L. Secco, G. Anania, N. Carbonaro, D. De Rossi, G. Magenes, "Heart Rate and Accelerometer Data Fusion for Activity Assessment of Rescuers During Emergency Interventions," IEEE Transactions on Information Technology in Biomedicine, vol.14, no.3, pp.702-710, May 2010.

[32] A Gaggioli, G Pioggia, G Tartarisco, G Baldus, D Corda, P Cipresso, G Riva. A Mobile Data Collection Platform for Mental Health Research. Personal and Ubiquitous Computing 17: 2. 241-251 February 2013

[33] Riva G, Raspelli S, Pallavicini F, Grassi A, Algeri D, et al. (2010) Interreality in the management of psychological stress: a clinical scenario. Stud Health Technol Inform 154: 20-25

[34] Serino S, Cipresso P, Gaggioli A, Riva G (2013). The Potential of Pervasive Sensors and Computing for Positive Technology: The Interreality Paradigm. In: Pervasive and Mobile Sensing and Computing for Healthcare, SSMI 2. Edited by: Mukhopadhyay S C et al.. p. 207232, Springer

[35] Riva G, Gaggioli A, Grassi A, Raspelli S, Cipresso P, et al. (2011) NeuroVR 2--a free virtual reality platform for the assessment and treatment in behavioral health care. Stud Health Technol Inform 163: 493-495. 